\title{
BERKELEY: ARGUMENTACIÓN FILOSÓFICA *
}

\author{
José A. ROBLES \\ Instituto de InVEstigaciones Filosóficas \\ Universidad Nactonal Autónoma de MÉxico
}

\section{Introducción}

Berkeley ha sido justamente alabado por la agudeza de sus argumentos pero, también, debido a esto, se le llegó a considerar un mero sofista, en un sentido despectivo - platónico_ del término - Boswell al expresar las opiniones de Samuel Johnson - o como un escéptico - a pesar de las múltiples afirmaciones contrarias que él mismo expresara- en la versión de Hume, de quien es la siguiente cita:

... en efecto, la mayoría de los escritos de ese autor tan ingenioso [Berkeley] forman las mejores lecciones de escepticismo que hayan de encontrarse sea entre los filósofos antiguos o los modernos, sin exceptuar a Bayle... que todos sus argumentos [los de Berkeley] son, en realidad, meramente escépticos, aunque se pretendiera otra cosa, lo muestra esto, que no admiten respuesta alguna y no producen convicción. $\mathrm{Su}$ único efecto es causar esas momentáneas sorpresas, indecisión y confusión que es el resultado del escepticismo. (Enq. Conc. Hum. Unders. XII, párr. 122, n.)

La opinión acerca de la obra de Berkeley ha ido cambiando con el tiempo y actualmente se le considera no sólo un argumentador brillante, sino además profundo. Lo que deseo hacer en las siguientes páginas es

- El presente escrito, del cual publiqué una versión resumida en la Revista Latinoamericana de Filosofía (de próxima publicación), con el título "Berkeley: Argumentación filosófica", surgió como un producto derivado de las reuniones del Grupo de estudios berkeleyanos que integramos en el Instituto de Investigaciones Filosóficas (UNAM), a fin de preparar un conjunto de charlas en conmemoración del tricentenario del nacimiento de Berkeley (12 de marzo de 1685). El tema de este artículo tuvo como disparador inmediato la cita de un texto de Popper (de su perceptivo artículo "A Note on Berkeley as Precursor of Mach and Einstein") que hiciera Juan Vázquez Abad en su charla dentro del ciclo conmemorativo. Aquí quiero agradecer a los integrantes del grupo el magnifico ambiente de trabajo que propiciaron en las reuniones y debido al cual pudimos llevar a cabo gratas y muy fecundas sesiones de comentarios y críticas a fondo de los diversos escritos que allí se leyeron antes de su presentación en el mencionado ciclo. En particular, quiero agradecer a Laura Benitez y a Carmen Silva sus agudas y muy pertinentes observaciones que me 
presentar la estructura de un argumento que Berkeley emplea para atacar objetivos diversos pero que, según él lo ve, caen bajo la misma caracterización de 'confusión lingǘstica'. Ciertamente a Berkeley se le recuerda, de manera central, como a) el crítico de las ideas abstractas lockeanas y b) el crítico de la idea de sustancia material, también en la filosofía de Locke. Berkeley, en la 'Introducción' a los Principios hace explícita su creencia de que una gran cantidad de errores en nuestra forma de pensar acerca del mundo surge por una concepción equivocada del lenguaje; él considera que la tesis de las ideas abstractas de Locke sólo puede surgir al tener una visión equivocada acerca de lo que éste es.

Mi interés en este análisis es poner de manifiesto la gran extensión de terreno que Berkeley cubre con su argumento lingüístico. Berkeley emplea el argumento para atacar la tesis lockeana de las ideas abstractas, pero también vuelve a aparecer en su ataque a los matemáticos, al rechazar la propuesta de éstos de que los objetos finitos de nuestro mundo perceptual están compuestos de un número infinito de partes o bien de que el análisis matemático se funda en tesis infinitesimalistas. Por otra parte, también lo aplica en filosofía natural, en la que rechaza la tesis de que los físicos tengan como finalidad ( $q u a$ físicos) llegar al conocimiento de la naturaleza interna (esencial) de los objetos del mundo exterior. Lo que considero importante de esta investigación es que nos permitirá adquirir una idea más clara y mejor de la dependencia o independencia que mantienen muchas de las tesis de Berkeley con respecto a su princiipo básico esse est percipi; lo anterior nos permitirá, por eso mismo, tener una visión más objetiva de su filosofía y saber qué tesis berkeleyanas permanecerán incólumes en caso de que sean efectivos los ataques que se hagan en contra de su principio.

Finalmente, quiero subrayar que en este escrito no deseo comprometerme, de manera primordial, con una evaluación de la verdad o no de algunas de las tesis de Berkeley, sino que tan sólo deseo considerar su aspecto estructural y la agudeza que tuvo su autor al formularlas.

\section{Estructura del argumento berkeleyano}

En la formulación de sus propuestas críticas, podemos encontrar, en los han aclarado tanto propuestas de Berkeley como de su muy ilustre y respetado antecesor: John Locke.

La elaboración del presente artículo se llevó a cabo empleando una microcomputadora Columbia, con software Perfect Writer, en la Dirección de Cómputo para la Investigación (DCI) de la UNAM. Le agradezco al director de esta dependencia universitaria, ingeniero Carlos Strassburger, la muy amistosa y siempre cordial disposición que ha tenido para facilitar mi labor en las instalaciones a su cargo. 
textos de Berkeley, un esbozo de tesis, como la que formulo de inmediato y que según su manera de ver las cosas, es la que ha producido muchos errores en la filosofía. La tesis que ahora presento, y que denominaré la 'falacia descriptivista', está compuesta de un supuesto filosófico básico, que expreso como el supuesto 2) en la formulación que sigue —este supuesto es el que Berkeley atacará de manera central-; el supuesto i) es un enunciado que, con cierta facilidad, podemos fundar en nuestra experiencia como usuarios conscientes del lenguaje.

\subsection{La falacia descriptivista}

1) Los signos lingüísticos, mediante los cuales hablamos acerca del mundo, tienen ciertas características semánticas que son las que nos permiten usarlos de la manera como lo hacemos - por ejemplo, hay términos como 'casa', 'animal', 'hombre', etcétera, que tienen la propiedad de ser generales; otros señalan a uno y sólo un individuo particular; otros nos remiten a aspectos o cualidades de los objetos, como 'grande', 'rojo', etcétera,

\section{y}

2) Para explicar (dar cuenta de) algunas propiedades semánticas de los signos lingüísticos se postulan ciertas entidades con las características que se consideran adecuadas para esto; o bien se cree que porque pueden efectuarse algunos manejos a nivel de signos, éstos siempre han de 'reflejarse' en la realidad.

Berkeley critica el supuesto 2) subrayando que es plausible mantenerlo sólo si suponemos que el lenguaje tiene una función única y que de ésta se derivan todas sus propiedades semánticas; la función única del lenguaje a la que aquí aludo sería la de "comunicar ideas" como lo expresara Berkeley ${ }^{1}$ o la de dar una descripción del mundo. Por esta razón he denominado 'falacia descriptivista' al error que Berkeley cree detectar en matemáticos, físicos y filósofos que mantienen implícita o explficitamente la tesis expresada en 2). La propuesta de Berkeley es la tesis que subraya que la dimensión del significado es mucho más amplia que la mera descripción; lo que considero que puede surgir de la propuesta de Berkeley es la tesis que señala que porque una palabra $\mathrm{X}$ es significativa es por lo que puede servir (usarse) para describir la

1 Cf. Principios, Introducción, secciones 18.19; véase, además, el Alciphron VII, 14, donde la tesis se vuelve a expresar. 
realidad. La función descriptiva del lenguaje no agota, sino que presupone la significatividad del mismo.

\section{Primer ejemplo: ideas abstractas}

Berkeley, en los Principios, Introducción, sección 11, nos dice:

La razón que aquí se asigna [Locke en Ensayo II, xi, 101] por la que no tenemos fundamento para creer que los brutos tengan ideas abstractas es que no observamos que ellos usen palabras $u$ otros signos generales cualesquiera y esto se basa en esta suposición, a saber, que hacer uso de palabras implica tener ideas generales. De lo cual se sigue que los hombres que usan el lenguaje son capaces de abstraer o de generalizar sus ideas. Que éste es el sentido y argumentación del autor se verá mejor por la respuesta que da a la pregunta que formula en otro lugar. 'Puesto que todas las cosas que existen son sólo particulares, ¿cómo llegamos a los términos generales? Su respuesta es, 'Las palabras se hacen generales al hacerlas los signos de ideas generales' [Ensayo L. 3, C. 3, Sec. 6]. Pero parece que una palabra se hace general al hacerla el signo, no de una idea abstracta general, sino de varias ideas particulares, sugiriendo cualquiera de ellas el término a la mente de manera indiferente...

La tentación que Berkeley cree percibir en los teóricos del lenguaje -en Locke de manera particular - es la tendencia a buscar una entidad dondequiera que en el lenguaje figure un sustantivo; por otra parte, si el sustantivo es, además, un término general, la entidad que tenga como contraparte tendrá que ser también general:

... se piensa que todo nombre tiene o debe de tener sólo una significación precisa y establecida, lo que inclina a los hombres a creer que hay ciertas ideas abstractas determinadas que constituyen la verdadera y única significación inmediata de cada nombre general (Principios, introducción, 18).

Más adelante, en los mismos Principios, señala de manera categórica:

... Aun cuando, en efecto, estamos tentados a creer que cada término sustantivo representa una idea distinta que puede separarse de todas las demás, lo que ha ocasionado errores sin fin ... (Principios I, 116).

Finalmente, en una de las anotaciones no publicadas de su 'Primera redacción [first draft] de la "Introducción"' a los Principios, se encuen- 
tra la propuesta más acabada en el sentido que aquí me interesa poner de manifiesto:

Otra cosa que hace que se crea que las palabras y las ideas son algo más inseparables de lo que lo son en verdad, es la opinión de que cada nombre representa una idea. Ahora, no nos debe sorprender que los hombres se fatiguen en vano y encuentren que es una tarea muy difícil cuando intentan descubrir y ver las ideas a las que apuntan esas palabras que, en verdad, no apuntan a ninguna. ${ }^{2}$

El argumento que Berkeley esgrime, de manera implícita, en el texto anterior, puede formularse en términos de la siguiente pregunta: "२Por qué hemos de concluir, a partir de propiedades inherentes a los signos, propiedades o entidades en el mundo?" Lo que Berkeley muestra en su formulación es una extrañeza acerca de la conclusión lockeana; de manera abreviada, el proceso inferencial puede formularse de esta manera: Porque hay signos generales [dato experimental], debe de haber entidades generales. Puesto que todos los individuos concretos de la experiencia son particulares [nuevamente, dato de experiencia que señala Locke y que Berkeley acepta], las entidades generales deben ser abstractas.

¿Qué supuestos acerca de la relación lenguaje-mundo están presentes en la argumentación de Locke? Puede haber muchos y muy diversos supuestos en la mente de Locke que no me interesa precisar; hay uno, sin embargo, que parece surgir, de manera clara, en la formulación que a Berkeley le interesa presentar y éste es, justamente, la falacia descriptivista. Así se puede dar cuenta del paso inferencial de la premisa de que hay signos generales a la conclusión de que debe de haber entidades generales, pues si un signo es general en su significación debe de haber, entonces, entidades generales en el mundo —entendiendo aquí por 'mundo', no tan sólo aspectos perceptuales del mismo, sino conceptuales, mentales, etcétera-, que son los que el signo 'refleja'.

La lección de Berkeley es pronto olvidada por los filósofos y el error que él señala surgirá nuevamente, con toda su fuerza, en las obras del primer Wittgenstein y de los atomistas lógicos. No será sino hasta las Investigaciones filosófcas de Wittgenstein, nuevamente, que se intentará ofrecer una terapia contra tal error, siguiendo, en algunos casos, las líneas que ya Berkeley apuntara a dos siglos de distancia.

\section{La falacia descriptivista y la matemática}

Un lugar ideal en el que Berkeley podía poner a prueba sus dotes 2 Works (Luce \& Jessop, eds.), II, p. 143. 
críticas era el escenario matemático de la época. La preocupación y el interés de Berkeley por los asuntos matemáticos están presentes desde la redacción de sus Comentarios filosóficos y la redacción y la lectura de un pequeño ensayo 'Acerca de los infinitos' ('Of Infinites') en los años de 1707-1708. Desde esa época temprana (Berkeley tenía entonces 22 años), encuentra en los escritos de los matemáticos muchos supuestos no fundados y alusiones a entidades curiosas -infinitesimales, infinitesimales de segundo orden o infinitesimales de infinitesimales, etcétera, que difícilmente podían resistir un mínimo escrutinio crítico.

Ahora bien, es justo decir que la matemática en los siglos xvir y xviri estaba sufriendo el mayor proceso de crecimiento y maduración que ha presenciado la historia $\mathrm{y}$, por esto, los matemáticos más destacados -entre ellos cito a Newton, los Bernoulli, Huygens, el mismo Leibniz, etcétera-, no tenían demasiado tiempo para sentarse a pensar y escribir acerca de los fundamentos de su ciencia. Los matemáticos de segundo y tercer niveles mucho hicieron para confundir a sus contemporáneos acerca de tales fundamentos. ${ }^{3}$ Así pues, Berkeley encuentra en los escritos de los matemáticos muchos motivos de queja $\mathrm{y}$, nuevamente, uno de ellos es que cometen la falacia descriptivista. Los dos ejemplos que presento aquí tienen una estructura similar, tan sólo que uno pertenece al campo de la geometría, el otro al del análisis.

\subsection{Segundo ejemplo: divisibilidad infinita.}

En los Principios I, sección 126, Berkeley nos dice:

En otro lugar he señalado que, en geometría, los teoremas y demostraciones se refieren a ideas universales. Sec. 15, Introd. $Y$ allí se explica en qué sentido ha de entenderse esto, a saber, que ha de suponerse que las líneas y figuras particulares incluidas en el diagrama representan otras innumerables de diferentes tamaños; o, en otras palabras, que el geómetra las considera abstraídas de su magnitud, lo que no debe implicar que él forme una idea abstracta, sino sólo que no le importa cuál sea la magnitud particular — sea ésta grande o

3 En general, algunos de los temas a los que aludo en esta sección y las subsiguientes los he tratado in extenso en mi libro en prensa: Las ideas matemáticas de George Berkeley, Obispo de Cloyne; algunos atisbos de las tesis que los matemáticos de los siglos xvi y xvin expresaron acerca de la matemática, los he presentado en 'Matemática hasta el siglo xvir. ¿Qué son los números?', en Diánoia 1982, pp. 155-171. Acerca de esto mismo véase, también, el libro clásico sobre fluxiones, de Cajori, $A$ History of the Conceptions of Limits and Fluxions in Great Britain, from Newton to Woodhouse (1919). 
pequeña - sino que la considera como algo que es indiferente para la demostración. Se sigue, por tanto, que de una línea en la demostración, que no tiene sino una pulgada de largo, debe hablarse como si contuviese diez mil partes, puesto que no se la considera en sí misma, sino como un universal y es universal sólo en su significación, por la cual representa innumerables líneas mayores que ella misma, en las que pueden distinguirse diez mil partes o más, aunque en ella no haya más de una pulgada. De esta manera, las propiedades de las líneas significadas se transfieren (mediante una figura muy usual) al signo y asi, por error, se cree que le pertenecen por propia naturaleza.

En este ejemplo podemos detectar, con toda claridad, la estructura del argumento berkeleyano: la conclusión que los matemáticos de la época (siglos xvir y xviri) extraen es que en una línea física - por pequeña que ésta sea- hay un número infinito de partes.

Una forma de alcanzar la conclusión señalada podría ser teniendo en cuenta la solución del siguiente problema:

Dada una recta $A B$, muestre cómo obtener, geométricamente, un segmento de longitud $A B / 2^{n}$.

La solución del problema anterior podría ofrecerse en los siguientes términos:

A. Trácese una recta de, por ejemplo, 2 pulgadas de largo en el pizarrón; sea ésta la recta $C D$.

1o. Si $n=0$, entonces sea

$$
\begin{aligned}
C D & =A B / 2^{\circ} \\
& =A B / 1 \\
& =A B .
\end{aligned}
$$

2o. Si $n>0$, supongamos que la recta $C D=A B / 2^{n-1}$; queremos obtener de ella un segmento $=A B / 2^{n}$.

Aplíquese a $C D$ el procedimiento para dividir una recta en mitades mediante el empleo del compás. El resultado será tener dos segmentos de recta de longitud $C D / 2$. Tómese cualquiera de estos segmentos y será el segmento con la longitud deseada, pues:

4 Berkeley alude a este ejemplo en Princ., Intr., 12. 


$$
\begin{aligned}
C D / 2 & =\left(A B / 2^{n-1}\right) / 2 \\
& =A B /\left(2^{n-1 \cdot 2}\right) \\
& =A B / 2^{n} .
\end{aligned}
$$

Q.E.D.

Ahora bien si, como nos dice Berkeley, los matemáticos transfieren al signo las propiedades de los objetos por él representados, dejarían de ver la recta $C D$ como signo o representante de cualesquiera otras rectas y concluirían que, por grande que fuera la $n$, de la recta misma $C D$ podrían obtenerse segmentos de longitud $C D / 2^{n}$.

Una proposición clásica, que figura en los Elementos de Euclides (X, prop. 1), es la conocida con el nombre de 'principio (o axioma) de (Eudoxo-)Arquímedes' que dice lo siguiente:

Dadas dos magnitudes desiguales, si de la mayor se sustrae una magnitud mayor que su mitad y si este proceso se repite continuamente, quedará alguna magnitud que será menor que la menor magnitud dada.

Traduzco la versión de Heath de la demostración.

Sean $A B$ y $C$ las dos magnitudes desiguales, de las que $A B$ es la mayor.

Digo que si a $A B$ se le quita una magnitud mayor que su mitad y a lo que queda se le quita una magnitud mayor que su mitad y si este proceso se repite continuamente, quedará alguna magnitud que sea menor que la magnitud $\mathrm{C}$.

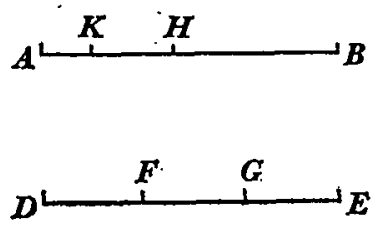

Pues si se multiplica $C$ será, en algún momento, mayor que $A B$. Mulplíquese $C$ y sea $D E$ un múltiplo de $C$ y mayor que $A B$. Divídase $D E$ en las partes $D F, F G, G E$, iguales a $C$; de $A B$ quítese $B H$, mayor que su mitad y de $A H$ quítese $H K$, mayor que su mitad. Repitase este proceso continuamente hasta que haya tantas divisiones en $A B$ como hay en $D E$. Sean, entonces, $A K, K H, H B$ tantas divisiones como $D F, F G, G E$.

Ahora bien, puesto que $D E$ es mayor que $A B$ y a $D E$ se le ha quitado 
$E G$, menor que su mitad, y de $A B$ se ha quitado $B H$, mayor que su mitad, entonces el remanente $G D$ es mayor que el remanente $H A$. Y, puesto que $G D$ es mayor que $H A$ y de $G D$ se ha quitado la mitad $G F$ y de $H A$ se ha quitado $H K$, mayor que la mitad, entonces el remanente $D F$ es mayor que el remanente $A K$. Pero $D F$ es igual a $C$.

Por tanto, de la magnitud $A B$ queda la magnitud $A K$ que es menor que la magnitud menor de la que partimos, a saber, $C$.

Q. E. D.

Acerca de la anterior proposición se puede decir lo mismo que de la primera; los matemáticos concluirian que esta propuesta es posible llevarla a cabo indefinidamente, con lo que se infiere que cualquier magnitud puede dividirse ad infinitum, la que es una proposición clásica de Euclides en sus Elementos; Berkeley replicaría, una vez más, sin embargo, que en esto se esconde la confusión señalada entre propiedades del signo considerado como universal y propiedades del objeto concreto del que se lleva a cabo la demostración.

\subsection{Tercer ejemplo: los fundamentos del cálculo}

En el 'Prefacio' que Bernard le Bouvier de Fontenelle (1657-1757) le escribe al libro Analyse des infiniment petits pour l'intelligence des lignes courbes (1696), de Guillaume François Antoine, Marquis de l'Hospital (1661-1704), podemos leer la siguiente exaltada caracterización del nuevo cálculo (el libro de de l'Hospital es el primer manual de cálculo escrito siguiendo los lineamientos de Leibniz y de los Bernoulli, en el que se considera sólo la porción diferencial en versión infinitesimalista):

El análisis que se explica en esta obra supone el [análisis] común, pero es muy diferente. El análisis ordinario sólo trata magnitudes finitas; éste penetra hasta el mismo infinito y compara las diferencias infinitamente pequeñas entre magnitudes finitas; descubre las razones de estas diferencias y, por esto, da a conocer las de las magnitudes finitas, las que, comparadas con las infinitamente pequeñas, son como algo infinito. Igualmente se puede decir que este análisis va más allá del infinito, pues no se detiene en las diferencias infinitamente pequeñas, sino que descubre las razones entre las diferencias de estas diferencias, así como también de las diferencias tercera, cuarta y así sucesivamente, sin jamás encontrar un término que lo pueda detener. De tal manera que este análisis no abarca tan sólo el infinito, sino el infinito del infinito o una infinitud de infinitos. 
Berkeley, acerca de los matemáticos y de sus pretensiones, escribió en The Analyst, sección 8, lo siguiente:

... Ellos [los matemáticos] no tienen escrúpulos para decir que con la ayuda de esta nueva analfícica [el cálculo infinitesimal de los matemáticos del continente europeo o el fluxionario de los ingleses seguidores de Newton] pueden penetrar el infinito mismo; que pueden extender su vista más allá del infinito; que su arte abarca no sólo el infinito, sino el infinito del infinito (como ellos lo expresan) o una infinitud de infinitos. Pero, a pesar de estas afirmaciones y pretensiones, puede justamente ponerse en duda si, como a otros hombres en otras investigaciones con frecuencia los engañan las palabras o los términos, ellos, de igual manera, no son maravillosamente defraudados y engañados por sus propios y peculiares signos, símbolos o especies. Nada es más fácil que idear expresiones o notaciones para fluxiones e infinitesimales de primero, segundo, tercero, cuarto y órdenes subsecuentes procediendo de la misma forma regular sin fin o límite

. .. $\therefore:$ :

x. x. x. x., etcétera, o bien $d x$. ddx. dddx. ddddx., etcétera...

Por otra parte, en ibid., sección 36, añade:

Con demasiada frecuencia los hombres se engañan a sí mismos y a otros como si concibiesen y entendiesen cosas expresadas mediante signos cuando, en verdad, no tienen idea alguna, salvo tan sólo la de los mismos signos...

En estas observaciones Berkeley critica agudamente a sus contemporáneos matemáticos pues, si bien es cierto que técnicamente impulsaron su actividad a grandes alturas, también es cierto que teóricamente tenían muchas oscuridades en lo tocante a los fundamentos de su labor y proponían tesis que se contradecían abiertamente unas a otras. Ciertamente, los matemáticos no tenían ninguna idea clara acerca del sistema de números que estaban manejando ${ }^{5}$ y mezclaban, sin rubor, infinitesimales con números reales; por otra parte, sus demostraciones carecian de rigor y con cierta facilidad emitian tesis ontológicas que no tenían ningún respaldo adecuado. Al igual que en los casos anteriores, es claro que en el actual Berkeley esgrime su navaja crítica señalándoles a los matemáticos la comisión de la falacia descriptivista. No deseo detenerme mucho en la consideración de este caso, ya que en otro lugar he dado mayores

5 Véase mi artículo 'Matemática hasta el siglo xviII. ¿Qué son los números?'; $c f$. supra n. 3. 
detalles sobre la crítica berkeleyana a los infinitesimales. ${ }^{6}$ Aquí presentaré, tan sólo, un ejemplo ilustrativo de argumentación matemática de la época y dejo al lector libre de emitir el juicio que mejor le plazca. La cita que viene a continuación es de un matemático de la época, Charles Hayes (1678-1760): ${ }^{\text {r }}$

La magnitud es divisible in infinitum y las partes, tras esta división infinita, siendo infinitamente pequeñas, son lo que los analistas denominan momentos, o diferencias; y si consideramos la magnitud como indeterminada y como creciendo o decreciendo perpetuamente, entonces el incremento o el decremento infinitamente pequeño se denomina la fluxión de esta magnitud o cantidad. Y sea que se les denomine momentos, diferencias o fluxiones, aún se supone que los mismos mantienen la misma proporción con sus totalidades, como un número finito la mantiene con uno infinito o como un espacio finito la mantiene con uno infinito. Ahora bien, siendo extensas esas partes infinitamente pequeñas, las mismas son infinitamente divisibles una vez más y estas partes infinitamente pequeñas de una parte infinitamente pequeña de una cantidad dada, las denominan los geómetras Infinitesimæ Infinitesimamorum o Fluxiones de Fluxiones. Asimismo, puede concebirse que una de esas partes infinitamente pequeñas se divida en un número infinito de partes que se denominan Terceras Fluxiones, etcétera.

5. Cuarto ejemplo: causalidad.

En los Principios I, 103, Berkeley señala:

El gran principio mecánico ahora en boga es la atracción. A muchos puede parecerles suficientemente explicado mediante ella el que una piedra caiga hacia la tierra o que el mar crezca hacia la luna...

Y más adelante (en ibid. sección 106), continúa:

Pero hemos de proceder con cuidado en tales cosas, pues somos capaces de darles demasiado peso a las analogías $y$, en perjuicio de la verdad, dejarnos llevar por esa avidez de la mente que la mueve a ampliar su conocimiento en teoremas generales. Por ejemplo, algunos están dispuestos a declarar universal la gravitación o atracción mutua porque aparece en muchos casos, y que el atraer y ser atraido por

6 En mi libro en prensa, véase supra n. 3.

7 El texto se encuentra en su libro $A$ Treatise of Fluxions: or An Introduction to Mathematical Philosophy (Londres, 1704); el mismo lo cita Cajori en op. cit., p. 42. 
cualquier otro cuerpo es una cualidad esencial, inherente a cualesquier cuerpos que sean...

En el De Motu, secciones 17, 18 y 28, respectivamente, nos dice Berkeley

Fuerza, gravedad, atracción y términos similares, son útiles en los razonamientos y en los cálculos acerca del movimiento y de los cuerpos en movimiento, pero no para entender la naturaleza simple del movimiento mismo o para indicar otras tantas cualidades diferentes...

Una explicación similar ha de darse de la composición y resolución de cualesquiera fuerzas directas en oblicuas mediante la diagonal y los lados del paralelogramo. Sirven al propósito de la ciencia mecánica y del cálculo; pero una cosa es servir para el cálculo y las demostraciones matemáticas y otra es presentar la naturaleza de las cosas.

Se dice que la acción y la reacción están en los cuerpos y esa forma de hablar se encuadra dentro de los propósitos de las demostraciones mecánicas; pero, debido a eso, no debemos suponer que haya en ellos alguna virtud real que es la causa o principio del movimiento. Pues esos términos han de entenderse de la misma manera que el término atracción; y aś como la atracción es tan sólo una hipótesis mecánica y no una cualidad física, lo mismo debe entenderse, también, de la acción y la reacción, y por la misma razón...

Junto a estos pasajes, en los que Berkeley nos habla de los objetos físicos, quiero añadir otros en los que su preocupación son los signos. Las primeras dos citas las tomo de los Comentarios filosóficos y éstan son:

Mo La razón por la que demostramos tan bien acerca de signos es $\mathrm{x}$ que son perfectamente arbitrarios $\mathrm{y}$ están en nuestro poder, hechos a placer. (CF 732)

Y dos anotaciones más adelante, añade:

Mo Muéstreme cualquier Hombre una Demostración no verbal $x \quad$ que no dependa, ya sea de algún principio falso o a 10 más de algún principio de la Naturaleza que es el efecto de la voluntad de Dios y no sabemos cuán pronto pueda cambiarse. (CF 734)

Algunos años después, en 1732, Berkeley publica su libro en forma de diálogo, Alciphron, y en él, Eufranor, el portavoz de Berkeley en esta obra, vuelve a expresar la doctrina diciendo: 
Si no me equivoco, se encontrará que todas las ciencias, en tanto son. universales y demostrables por la razón humana, tienen signos como su objeto inmediato, aun cuando a éstos se les refiere a cosas en su aplicación ... (Diál. VII, 13)

La distinción que aquí establece Berkeley con toda nitidez es la que mantenemos contemporáneamente entre ciencias factuales y ciencias formales. Esta precisa y radical distinción le permite, asimismo, mostrar, de manera muy clara, cómo, vía la falacia descriptivista, se cometen graves errores en el caso de la filosoffa natural o física de la época: Si 1. la demostración (con carácter de necesidad) se da a nivel de signos y 2. se acepta la tesis descriptivista entre signo y realidad, los filosofos naturales (físicos) concluyen que en la naturaleza se dan relaciones necesarias entre los objetos físicos.

En este caso el dispositivo crítico berkeleyano funciona con brillo extremo: dado que no tenemos fundamento para aceptar la premisa 2, en el argumento anterior, no tenemos conclusión, por lo que los filósofos naturales nos deben una prueba de que hay necesidad en la naturaleza. Nuestra visión contemporánea de la ciencia jsigue manteniendo la propuesta berkeleyana!

Otra confusión que trae aparejada la anterior, es concluir que la filosofía natural (la física) descubre la naturaleza íntima (esencial) de los objetos de la naturaleza cuando, en realidad, lo único que puede hacer es descubrir regularidades en el comportamiento de tales objetos. Lo que los físicos pueden ofrecernos son algoritmos que nos permiten predecir el comportamiento de los objetos del mundo exterior, pero de esto no se sigue que, por ello, estemos descubriendo su esencia. Berkeley ve la ciencia, asi, como una empresa descriptiva, mas no explicativa o especulativa (como él solía decirlo). Para él el verdadero conocimiento de las cosas, de su naturaleza misma, era la competencia, no de la física, sino de la metafísica.

\section{Conclusión.}

En el De Motu, 1, Berkeley asentó:

En la búsqueda de la verdad debemos siempre cuidarnos de que no nos engañen los términos que no entendemos correctamente. Esta es la propuesta principal. Casi todos los filosofos emiten la advertencia; pocos la observan.

Berkeley no tan sólo emite la advertencia sino que su filosofía gira en torno a las trampas y asechanzas lingüísticas y nuestro autor siempre está 
apuntando posibles peligros que surgen por malos usos del lenguaje o bien por mantener tesis inadecuadas acerca de la relación lenguajemundo. Los casos que he presentado en estè escrito son, precisamente, casos en los que se violenta la relación lenguaje-mundo y se desean establecer fuertes nexos descriptivistas entre el lenguaje y la realidad. Berkeley tuvo clara conciencia de que no siempre la relación entre lenguaje y mundo es de descripción, o bien, no siempre es la forma más útil de interpretar la relación lenguaje-mundo, cuando realmente existe alguna relación así.

Lo que Berkeley descubre, analiza y condena es justamente ese confundir usos y relaciones lingüisticos que llevaban a sus contemporáneos, tanto científicos como filósofos, a cosificar expresiones lingüísticas, más que a descubrir entidades con algún tipo de realidad en el mundo.

En los ejemplos que aquí he presentado de discusión berkeleyana se pueden descubrir vetas de argumentación filosófica contemporánea. Visto desde cierta perspectiva, el ataque de Berkeley a las ideas abstractas lockeanas tiene todos los visos de ser una propuesta antiesencialista, con lo que - junto con la tesis de considerar la significatividad de las palabras en términos de contextos de uso- se podría ver a Berkeley como un claro antecesor de Wittgenstein. ${ }^{8}$ Por otra parte, creo que es justo ver la posición de Berkeley, con respecto a ciertas porciones de la matemática, dentro del marco de la posición formalista contemporánea; ${ }^{\ominus}$ por último, las propuestas de Berkeley en física lo presentan como un precursor de los instrumentalistas en esta disciplina. El siguiente pasaje es una muestra más de la posición que mantuvo Berkeley tanto ante la f́sica como ante la matemática:

$\mathrm{Y}$ así como los geómetras, debido a su arte, emplean muchos artificios que ni ellos mismos pueden describir ni encontrar en la naturaleza de las cosas, de igual manera el mecánico [mechanician] emplea ciertos términos abstractos y generales, imaginando en los cuerpos fuerza, acción, atracción, solicitación, etcétera, que son de gran utilidad para las teorías y las formulaciones, así como también para los cómputos acerca del movimiento, aun si en la verdad de las cosas y en los cuerpos que realmente existen se las buscaría en vano, tal como las ficciones de los geómetras hechas por abstracción matemática. (De Motu, 39.)

8 Sin embargo, las dudas que tengo acerca de esto - con lo que me uno a la posición crítica de Bennett en su libro Locke, Berkeley, Hume- las expreso en otro texto: 'Locke y Berkeley: abstracción y generalidad' que lef en una sesión del Seminario de Investigadores de nuestro Instituto.

- Esta propuesta la he intentado fundamentar en mi libro; cf. supra n. 3. 
Finalmente, cabe señalar que la discusión en el contexto berkeleyano que aquí he considerado, tiene la doble virtud de mostrarnos, a) cómo un mismo argumento puede ser fértil y emplearse en campos muy diversos y b) la agudeza de su autor quien, por medio de sus observaciones, nos da muestra de una argumentación hábil y certera y, con ella, además de causarnos sorpresa - para volver mínimamente a Hume-, en muchas ocasiones nos permite obtener una comprensión mejor del mundo o bien, poner a prueba nuestra capacidad lógica para defender nuestros más caros prejuicios de los ataques provenientes de su pluma. 